\title{
ESPACES FIBRÉS LINÉAIRES FAIBLEMENT NÉGATIFS SUR UN ESPACE COMPLEXE $\left({ }^{1}\right)$
}

PAR

\author{
VINCENZO ANCONA
}

ABSTRACT. Let $F$ be a coherent sheaf over a compact reduced complex space $X, L(F)$ the linear fibre space associated with $F, S^{k}(F)$ the $k$ th symmetric power of $F$. We show that if the zero-section of $L(F)$ is exceptional, then $H^{r}\left(X, E \otimes_{O X} S^{k}(F)\right)=0$ for every coherent sheaf $E$ on $X$ and for $r>1$ and sufficiently large $k$. Using this result, we deduce moreover that Supp $F$ is a Moišezon space.

Introduction. Dans [6] H. Grauert introduit la notion de négativité faible pour un espace fibré linéaire (non nécessairement localement trivial) sur un espace complexe, et dans le cas localement trivial démontre un critère de projectivité pour les espaces complexes compacts $[6$, Satz $2, \S 3]$ qui généralise celui de Kodaira [8].

Ici on donne une généralisation faible de ce critère dans le sens suivant. Etant donné un faisceau analytique cohérent sur un espace complexe compact, on dit qu'il est faiblement positif si l'espace linéaire associé est faiblement négatif au sens de Grauert; on montre donc qu'un espace complexe compact réduit $X$ qui porte un faisceau $F$ faiblement positif tel que Supp $F=X$ est un espace de Moišezon (Théorème 4.1).

La preuve utilise le théorème, dû à Rossi [12], que pour tout faisceau cohérent $S$ sur $X$ on peut trouver une modification propre $\pi: \widetilde{X} \rightarrow X$ telle que le faisceau $\widetilde{S}$, quotient de $\pi^{*} S$ par son sous-faisceau de torsion, soit localement libre. Si l'on pouvait démontrer que si $S$ est faiblement positif $\widetilde{S}$ l'est aussi, le Théorème 4.1 serait une conséquence immédiate du résultat de Grauert. Malheureusement on est loin d'une telle conclusion, parce qu'on ne voit pas bien les rapports entre les espaces fibrés linéaires associés aux faisceaux $S$ et $\widetilde{S}$.

Pour démontrer le Théorème 4.1 on suit alors le procédé de Grauert: on établit un théorème d'annulation de la cohomologie (Théorème 3.2) graĉe auquel (et en faisant appel seulement à ce moment au résultat de Rossi) on peut

Received by the editors November 20, 1973.

AMS (MOS) subject classifications. Primary 32C35, 32J99, $32 \mathrm{~L} 99$.

Key words and phrases. Puissance symétrique, espace fibré linéaire faiblement négatif, faisceau faiblement positif, espace de Moišezon.

(1) The present paper was written while the author was a member of G. N. S. A. G. A. (C. N. R.). 
trouver un'application biméromorphe (au sens de Remmert [9]) de $X$ sur un sous-espace analytique d'une grassmannienne.

Le Théorème 4.1 est voisin de certains résultats obtenus par Riemenschneider [10], [11].

Le $\S 1$ de ce travail contient des rappels et des préliminaires; dans le $\S 2$ on étudie en détail une filtration de certains groupes de cohomologie (Proposition 2.3); au $\S 3$ on établit le théorème d'annulation de la cohomologie (Théorème 3.2), aussi dans sa forme relative (Théorème 3.4); dans le $\$ 4$ on démontre finalement le Théorème 4.2, et un autre théorème (4.3) qui établit le même résultat sous des hypothèses différentes.

Les résultats de ce travail ont été annoncés dans [1].

Je remercie $G$. Tomassini, avec lequel $j$ 'ai eu des nombreuses discussions sur les arguments de ce travail.

1. Préliminaires.

1. Soient $X$ un espace complexe, $F$ un faisceau analytique cohérent sur $X$. Notons $S(F)$ l'algèbre symétrique de $F$; c'est un' $O_{X}$-algèbre graduée de présentation finie. Soit $S(F)=\bigoplus_{k \geqslant 0} S^{k}(F) ; S^{k}(F)$ est la $k$-ième puissance tensorielle symétrique de $F$; c'est un $O_{X}$-module cohérent.

Soit

$$
\mathrm{O} \rightarrow \mathrm{H} \rightarrow \mathrm{O}_{X}^{p} \rightarrow \mathrm{F} \rightarrow \mathrm{O}
$$

une résolution de $F$ sur un ouvert $U$ de $X$. En passant aux algèbres symétriques on obtient un'application surjective $\tilde{\alpha}: O_{X}\left[T_{1}, \ldots, T_{p}\right] \rightarrow S(F)$. Soit $J=$ $\operatorname{Ker} \tilde{\alpha}$. Le lemme suivant, bien connu, permet de décrire $J$ :

LEMme 1.1. Soit $A$ un anneau, et $0 \rightarrow K \rightarrow A^{p} \rightarrow M \rightarrow 0$ une suite exacte d'A-modules. On a alors la suite exacte d'algèbres sur $A: 0 \rightarrow N \rightarrow$ $A\left[T_{1}, \ldots, T_{p}\right] \rightarrow S(M) \rightarrow 0$ où $N$ est l'idéal de $A\left[T_{1}, \ldots, T_{p}\right]$ engendré par les formes linéaires $a_{1} T_{1}+\ldots+a_{p} T_{p}$ telles que $\left(a_{1}, \ldots, a_{p}\right) \in K$.

La graduation canonique de $O_{X}\left[T_{1}, \ldots, T_{p}\right]$ induit sur $J$ une graduation: on a $J=\bigoplus_{k>0} J_{k}$ où $J_{k}=J \cap S^{k}\left(O_{X}^{p}\right)$ et pour tout entier positif $k$ une suite exacte

$$
0 \rightarrow J_{k} \rightarrow S^{k}\left(O_{X}^{p}\right) \stackrel{\tilde{\alpha}_{k}}{\longrightarrow} S^{k}(F) \rightarrow 0 .
$$

Il s'ensuit que $J_{k}$ est un faisceau analytique cohérent, et le Lemme 1.1 implique que pour tout $x$ de $U, J_{k, x}$ est formé des polynômes homogènes en $T_{1}, \ldots, T_{p}$ à coefficients dans $O_{X, x}$ du type

$$
P\left(T_{1}, \ldots, T_{p}\right)=\sum_{i=1}^{l} f_{i}\left(T_{1}, \ldots, T_{p}\right)\left(a_{i 1} T_{1}+\ldots+a_{i p} T_{p}\right)
$$


où $f_{i}\left(T_{1}, \ldots, T_{p}\right) \in \mathcal{O}_{X, x}\left[T_{1}, \ldots, T_{p}\right], \operatorname{deg} f_{i}=k-1$ et $\left(a_{i 1}, \ldots, a_{i p}\right) \in H_{x}$. Soit maintenant

$$
0 \rightarrow \dot{H}^{\prime} \rightarrow O_{X}^{p^{\prime}} \stackrel{\alpha^{\prime}}{\longrightarrow} F \rightarrow 0
$$

un'autre résolution de $F$ sur $U$. Si $U$ est un ouvert de Stein une simple application du Theorème B montre qu'on a un diagramme commutatif (sur $U$ ):

$$
\begin{aligned}
& 0 \rightarrow H \rightarrow O_{X}^{p} \rightarrow F \rightarrow 0 \\
& \uparrow_{H} \rightarrow O_{X}^{p^{\prime}} \rightarrow F \rightarrow 0
\end{aligned}
$$

d'où on obtient un autre diagramme commutatif

$$
\begin{aligned}
& 0 \rightarrow J_{k} \rightarrow S^{k}\left(O_{X}^{p}\right) \rightarrow S^{k}(F) \rightarrow 0 \\
& \prod_{0} \rightarrow J_{k}^{\prime} \rightarrow S^{k}\left(O_{X}^{p^{\prime}}\right) \rightarrow S^{k}(F) \rightarrow 0
\end{aligned}
$$

2. Notons $Y=L(F)$ l'espace fibré linéaire associé à $F$ (v. [5]), et $\pi: L(F)$ $\rightarrow X$ la projection. Localement $L(F)$ se construit de la manière suivante: si $x \in X$ et $U$ est un voisinage ouvert de $x$ sur lequel on a la résolution (1) de $F$, on a un'immersion fermée de $V=L(F) \mid U\left({ }^{2}\right)$ dans $U \times C^{p}$ dont l'image est le sous-espace analytique fermé $V_{1}$ de $U \times \mathbf{C}^{p}$ défini par l'idéal $I$ de $O_{U \times c^{p}}$ engendré par les fonctions du type $a_{1} z_{1}+\ldots+a_{p} z_{p}$ avec $\left(a_{1}, \ldots, a_{p}\right) \in \Gamma(U, H)$ $\left(z_{1}, \ldots, z_{p}\right.$ étant les coordonnés dans $\left.\mathrm{C}^{p}\right)$. Si $\left(1^{\prime}\right)$ donne un'autre résolution de $F$ sur $U$, on obtient un'immersion fermée de $V$ dans $U \times \mathbf{C}^{p^{\prime}}$ d'image un sous-espace analytique fermé $V_{2}$ de $U \times \mathbf{C}^{p^{\prime}}$, et du diagramme (3) on tire un'application analytique $u^{\prime}: U \times \mathbf{C}^{p} \rightarrow U \times \mathbf{C}^{p^{\prime}}$ au dessus de $U$, linéaire sur les fibres, qui induit un isomorphisme de $V_{1}$ sur $V_{2}$, et tel que le diagramme suivant

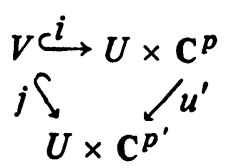

soit commutatif ( $i$ et $j$ indiquent les immersions décrites ci-dessus). Les fibres $L_{x}=\pi^{-1}(x)$ de $\pi$ sont des espaces vectoriels (réduits) de dimension égale à $\operatorname{dim}_{\mathbf{C}} F_{x} / m_{x} F_{x}\left(m_{x}\right.$ idéal maximal de $\left.O_{X, x}\right)$.

Une forme linéaire sur $Y=L(F)$ est un'application analytique $Y \rightarrow X \times \mathbf{C}$ au dessus de $X$ linéaire sur les fibres. Pour chaque ouvert $U$ de $X$ notons $F(L(F))(U)$ l'ensemble des formes linéaires sur $L(F) \mid U$. On obtient ainsi un

(2) Si $\pi: Y \rightarrow Z$ est un'application, et $Y^{\prime}, Z^{\prime}$ sont deux sous-ensembles de $Y$ et $Z$ respectivement, on pose $Y^{\prime} \mid Z^{\prime}=Y^{\prime} \cap \pi^{-1}\left(Z^{\prime}\right)$. 
faisceau analytique $F(L(F))$ sur $X$, et on a un isomorphisme canonique d' $O_{X^{-}}$ modules $F(L(F)) \simeq F($ v. [5]).

3. Soit $X$ un espace complexe, $F$ un faisceau analytique cohérent sur $X$. Pour tout entier positif $l$ l'ensemble des faisceaux quotients de $F$ qui sont localement libres de rang $l$ admet une structure d'espace complexe, noté Grass ${ }_{l}$ F (v. [4, exposé 12]); de cet espace nous utiliserons les propriétés suivantes. A toute surjection de faisceaux coherents $E \rightarrow F$ il correspond un'immersion fermée Grass $l$ F $\hookrightarrow$ Grass $_{l} E$. Pour $q>l$ on a Grass $l\left(O_{X}^{q}\right)=X \times G(q-l, q)$ où $G(q-l, q)$ est la grassmannienne des sous-espaces vectoriels de dimension $q-l$ de $\mathbf{C}^{q}$. Finalement si $E$ est un faisceau localement libre de rang $l$ sur $X$, on a Grass $l E=X$.

4. Soit $G$ un ouvert de $\mathbf{C}^{n}$ et $\varphi: G \rightarrow \mathbf{R}$ une fonction $C^{\infty}$. Soient $\left(z_{1}, \ldots, z_{n}\right)$ les coordonnés de $\mathrm{C}^{n}$ et $w \in G$. La forme hermitienne

$$
L(\varphi ; w)=\sum_{i, j} \frac{\partial^{2} \varphi}{\partial z_{i} \partial \bar{z}_{j}}(w) z_{i} \bar{z}_{j}
$$

s'appelle forme de Levi de $\varphi$ au point $w$. On dit que $\varphi$ est fortement pseudoconvexe sur $G$, si $L(\varphi ; w)$ est définie positive en tout point $w$ de $G$.

Si $X$ est un espace complexe, on dit qu'une fonction $\varphi: X \rightarrow \mathrm{R}$ est fortement pseudoconvexe si pour tout point $x \in X$ il existe un'immersion fermée $\tau$ d'un voisinage $U$ de $x$ dans un ouvert $G$ d'un espace affine, et une fonction $\phi$ : $G \rightarrow \mathbf{R}$ fortement pseudoconvexe, telle que $\varphi \mid U=\phi \circ \tau$.

Soit $\pi: X \rightarrow S$ un'application holomorphe entre espaces complexes. On dit (v. par exemple [7]) que $\pi$ est 1-convexe si pour tout $s \in S$ il existe un voisinage ouvert $U$ de $s$, une fonction $\varphi: X \mid U \rightarrow \mathbf{R}$ et une constante $c_{0} \in \mathbf{R}$ tels que:

(i) $\varphi \mid\left\{x \in X \mid U: \varphi(x)>c_{0}\right\}$ est fortement pseudoconvexe.

(ii) Pour tout $c \in \mathrm{R}$ l'application $\pi \mid\{x \in X \mid U: \varphi(x) \leqslant c\}$ est propre.

(iii) $\varphi \mid\left\{x \in X \mid U: \varphi(x)>c_{0}\right\}$ n'a que des minimums locaux isolés.

Dans le cas où $S$ est réduit à un point, on obtient les espaces fortement pseudoconvexes d'Andreotti-Grouert [2], que nous appellerons simplement espaces 1-convexes.

Si $\pi: X \rightarrow S$ est un'application holomorphe 1-convexe et $F$ un faisceau analytique cohérent sur $X$, les faisceaux images directes $R^{p} \pi_{*} F$ sont cohérents sur $S$ pour tout entier $p \geqslant 1 \quad$ [7, Theorem 3.5]).

5. Soit $\pi: \widetilde{X} \rightarrow X$ un'application holomorphe entre deux espaces complexes. On dit que $\pi$ est une modification propre si elle est propre et surjective, et il existe un sous-ensemble analytique maigre $N$ de $X$ tel que $\pi \widetilde{X} \backslash \pi^{-1}(N)$ soit un isomorphisme analytique de $\widetilde{X} \backslash \pi^{-1}(N)$ sur $X \backslash N$.

Nous aurons besoin du résultat suivant dû à Rossi [12] : 
THÉOREMÈ 1.2. Soient $X$ un espace complexe irréductible et $S$ un faisceau analytique cohérent sur $X$. Il existe alors un espace complexe irréductible $\widetilde{X}$ et une modification propre $\pi: \widetilde{X} \rightarrow X$ ayant les propriétés suivantes:

(i) Si $N=\mathbf{C}\left\{x \in X: S_{x}\right.$ est libre sur $\left.O_{X, x}\right\}$, alors $\pi \mid \widetilde{X} \backslash \pi^{-1}(N)$ est un isomorphisme de $\widetilde{X} \backslash \pi^{-1}(N)$ sur $X \backslash N$.

(ii) L'image inverse $S^{*}=\pi^{*} S$ de $S$ est un faisceau localement libre à moins de la torsion, i.e. si $T\left(S^{*}\right)$ est le sous-faisceau de torsion de $S^{*}, S^{*} / T\left(S^{*}\right)$ est localement libre.

6. Il est bien connu que si $\mathrm{X}$ est un espace complexe compact réduit et irréductible, le degré de transcedence sur $\mathbf{C}$ du corps des fonctions méromorphes globales sur $X$ est inférieur ou égal à $n=\operatorname{dim}_{\mathrm{C}} X$. S'il est exactement égal à $n$, on dit que $X$ est un espace de Moišezon. Si $X$ n'est pas irréductible, on dit qu'il est un espace de Moišezon si toute composante irréductible de $X$ l'est.

Tout sous-espace analytique réduit d'un espace projectif est de Moišezon; en effet par le théorème de Chow il est une variété algébrique. Il s'ensuit que tout espace complexe qui possède un'application biméromorphe (au sens de Remmert [9]) sur un sous-espace analytique d'un espace projectif est un espace de Moišezon.

Il est facile aussi de voir que si $\pi: \widetilde{X} \rightarrow X$ est une modification propre, $X$ est un espace de Moišezon si et seulement si $\widetilde{X}$ l'est.

2. Filtration de certains groupes de cohomologie.

1. Soient $X$ un espace complexe, $F$ un faisceau analytique cohérent sur $X, Y=L(F)$ l'espace fibré linéaire associé à $F$. Soit $U \subset X$ un ouvert de Stein, tel que sur $U$ on ait la suite exacte (1). A cette suite il correspond un'immersion fermée $V=L(F) \mid U \hookrightarrow U \times \mathbf{C}^{p}$ et pour tout entier positif $k$ une suite exacte (2), d'où, comme $U$ est de Stein, une suite exacte

$$
0 \rightarrow \Gamma\left(U, J_{k}\right) \rightarrow \Gamma\left(U, S^{k}\left(O_{X}^{p}\right)\right) \stackrel{\tilde{\alpha}_{k}}{\longrightarrow} \Gamma\left(U, S^{k}(F)\right) \rightarrow 0 .
$$

Soit $f \in \Gamma\left(V, O_{Y}\right)$. Comme $U \times \mathbf{C}^{p}$ est un espace de Stein, il existe $\widetilde{f} \in$ $\Gamma\left(U \times \mathbf{C}^{p}, O_{U \times \mathbf{C}^{p}}\right)$ dont la restriction à $V$ soit $f$. Développons $\widetilde{f}$ en série par rapport aux coordonnés $z_{1}, \ldots, z_{p}$ de $\mathbf{C}^{p}$ :

$$
\tilde{f}(x, z)=\sum_{i_{1}, \ldots, i_{p}} \widetilde{f}_{i_{1}, \ldots, i_{p}}(x) z_{1}^{i_{1}} \ldots z_{p}^{i_{p}}=\sum_{k} \widetilde{f}_{k}(x, z)
$$

$\operatorname{avec} \widetilde{f}_{i_{1}, \ldots, i_{p}} \in \Gamma\left(U, O_{X}\right)$ (pour l'existence et l'unicité d'un tel développement v. [2], [3]).

La fonction $\widetilde{f}_{k}=\Sigma_{i_{1}}, \ldots, i_{p} f_{i_{1}}, \ldots, i_{p} z_{1}^{i_{1}} \ldots z_{p}^{i_{p}}$ détermine une forme linéaire sur $U \times \mathbf{C}^{l}, l=\left(\begin{array}{c}k+p-1 \\ p-1\end{array}\right)$ : 


$$
U \times \mathrm{C}^{l} \rightarrow U \times \mathrm{C}, \quad\left(x, X_{k}^{i_{1}^{1}, \ldots, i_{p}}\right) \rightarrow \sum_{i_{1}+\ldots+i_{p}=k} \widetilde{f}_{i_{1}, \ldots, i_{p}}(x) X_{k}^{i_{1}, \ldots, i_{p}}
$$

où les $X_{k}^{i_{1}, \ldots, i_{p}}\left(i_{1}+\ldots+i_{p}=k\right)$ désignent les coordonnés de $\mathbf{C}^{l}$. Si l'on appelle encore $\widetilde{f_{k}}$ cette forme linéaire et on tient compte que $U \times \mathbf{C}^{l}=L\left(S^{k}\left(O_{X}^{p}\right)\right)$, on peut écrire $\widetilde{f_{k}} \in \Gamma\left(L\left(S^{k}\left(O_{X}^{p}\right)\right), F\left(L\left(S^{k}\left(O_{X}^{p}\right)\right)\right)\right)$.

Si $I_{k}$ désigne l'isomorphisme canonique entre $F\left(L\left(S^{k}\left(O_{X}^{p}\right)\right)\right)$ et $S^{k}\left(O_{X}^{p}\right)$, on obtient alors un élément $I_{k}\left(\widetilde{f_{k}}\right)$ de $\Gamma\left(U, S^{k}\left(O_{X}^{p}\right)\right)$ donné évidemment par:

$$
I_{k}\left(\tilde{f}_{k}\right)=\sum_{i_{1}+\ldots+i_{p}=k} \tilde{f}_{i_{1}, \ldots, i_{p}} T_{1}^{i_{1}} \ldots T_{p}^{i_{p}} .
$$

Posons $f_{k}=\widetilde{\alpha}_{k}\left(\mathcal{I}_{k}\left(\widetilde{f_{k}}\right)\right) \in \Gamma\left(U, S^{k}(F)\right)$. L'elément $f_{k}$ ainsi obtenu ne dépend ni du choix de la résolution de $F$ sur $U$ ni du choix de la fonction $\widetilde{f}$. Soit en effet ( $\left(1^{\prime}\right)$ un'autre résolution de $F$ sur $U$ et soit $\widetilde{f}^{\prime} \in \Gamma\left(U \times \mathbf{C}^{p^{\prime}}, O_{U \times C^{p^{\prime}}}\right)$ un'autre extension de $f$. On a un diagramme commutatif (5). Puisque la section nulle de $U \times \mathbf{C}^{p}$ est incluse dans $V$, on aura pour tout $x \in U:\left(\widetilde{f}^{\prime} \circ u^{\prime}-\widetilde{f}\right)_{(x, 0)} \in I_{(x, 0)}$ où $I$ est l'idéal de $O_{U \times \mathbf{C}} p$ qui définit $V$ dans $U \times \mathbf{C}^{p}$.

Il suffit alors de montrer le lemme suivant:

LEMME 2.1. Si $\tilde{f}_{(x, 0)} \in I_{(x, 0)}$, alors $\tilde{\alpha}_{k}\left(I_{k}\left(\widetilde{f}_{k, x}\right)\right)=0(k=0,1,2, \ldots)$.

Preuve. Supposons que $H_{x}$ soit engendré sur $O_{X, x}$ par les familles $\left(f_{i, 1}, \ldots, f_{i, p}\right)(i=1, \ldots, t)$ d'éléments de $O_{X, x}$. Alors $I_{(x, 0)}$ est engendré sur $O_{U \times C^{p},(x, 0)}$ par les fonctions du type $\sum_{j=1}^{p} f_{i j} z_{j}(i=1, \ldots, t)$. Sur un voisinage convenable de $(x, 0)$ dans $U \times \mathrm{C}^{p}$ il existe donc des fonctions holomorphes $g_{i}(y, z) \quad(i=1, \ldots, t)$ telles que

$$
\widetilde{f}(y, z)=\sum_{i=1}^{t}\left[g_{i}(y, z)\left(\sum_{j=1}^{p} f_{i j}(y) z_{j}\right)\right]
$$

d'où en développant chaque $g_{i}$ en série par rapport à $z_{1}, \ldots, z_{p}$ :

$$
\tilde{f}(y, z)=\sum_{i=1}^{t}\left[\left(\sum_{i_{1}, \ldots, i_{p}} g_{i_{i}, i_{1}, \ldots, i_{p}}(y) z_{1}^{i_{1}} \ldots z_{p}^{i_{p}^{p}}\right)\left(\sum_{j=1}^{p} f_{i j}(y) z_{j}\right)\right] .
$$

Il's'ensuit $\widetilde{f}_{0}=0$ et donc $\tilde{\alpha}_{0}\left(I_{0}\left(\tilde{f}_{0}\right)\right)=0$, et pour $k \geqslant 1$ :

$$
I_{k}\left(\widetilde{f}_{k, x}\right)=\sum_{i=1}^{t}\left[\left(\sum_{i_{1}+\ldots+i_{p}=k} g_{i, i_{1}, \ldots, i_{p}, x} T_{1}^{i_{1}} \ldots T_{p}^{i_{p}}\right)\left(\sum_{j=1}^{p} f_{i j, x} T_{j}\right)\right] .
$$

Compte tenu de la description de $J_{k, x}$ qui suit le Lemme 1.1, on a $\left.I_{k}\left(\widetilde{f}_{k, x}\right)\right) \in$ $J_{k, x}$ et donc $\widetilde{\alpha}_{k}\left(I_{k}\left(\widetilde{f}_{k, x}\right)\right)=0$. On peut donc définir, pour tout entier $k \geqslant 0$, un'application linéaire de C-espaces vectoriels: 


$$
\beta_{k}: \Gamma\left(V, O_{Y}\right) \rightarrow \Gamma\left(U, S^{k}(F)\right), \quad f \mapsto f_{k}
$$

Posons

$$
\Gamma\left(V, O_{Y}\right)_{k}=\left\{f \in \Gamma\left(V, O_{Y}\right): \beta_{0}(f)=\ldots=\beta_{k-1}(f)=0\right\} .
$$

On obtient une filtration de $\Gamma\left(V, O_{Y}\right)$ :

$$
\Gamma\left(V, O_{Y}\right)=\Gamma\left(V, O_{Y}\right)_{0} \supset \Gamma\left(V, O_{Y}\right)_{1} \supset \Gamma\left(V, O_{Y}\right)_{2} \supset \ldots
$$

et pour tout $k \geqslant 0$ une suite exacte

$$
0 \rightarrow \Gamma\left(V, O_{Y}\right)_{k+1} \rightarrow \Gamma\left(V, O_{Y}\right)_{k} \stackrel{\beta_{k}}{\longrightarrow} \Gamma\left(U, S^{k}(F)\right) \rightarrow 0
$$

qui est scindée; on peut en effet définir un'application linéaire $\sigma_{k}: \Gamma\left(U, S^{k}(F)\right) \longrightarrow$ $\Gamma\left(V, O_{Y}\right)_{k}$ telle que $\beta_{k} \circ \sigma_{k}=$ identité de la manière suivante. Soit $f_{k} \in$ $\Gamma\left(U, S^{k}(F)\right)$; à cause de l'exactitude de la suite (6) il se relève en un élément $\hat{f}_{k}$ de $\Gamma\left(U, S^{k}\left(O_{X}^{p}\right)\right)$ qui peut s'écrire, grâce à l'isomorphisme $I_{k}$, sous la forme $I_{k}\left(\widetilde{f_{k}}\right)$, avec $\widetilde{f}_{k} \in \Gamma\left(U \times \mathbf{C}^{p}, O_{U \times \mathbf{C}} p\right) ; \widetilde{f_{k}}$ est un polynôme homogène de degré $k$ en les variables $z_{1}, \ldots, z_{p}$. Il s'ensuit que sa restriction à $V$ appartient à $\Gamma\left(V, O_{Y}\right)_{k}$; nous posons alors $\sigma_{k}\left(f_{k}\right)=\widetilde{f}_{k} \mid V$. Pour montrer que cet élément est indépendant de la résolution choisie pour $F$ sur $U$ et du choix du relèvement $\hat{f}_{k}$, il suffit, compte tenu du diagramme (4), de prouver le lemme suivant:

LEMME 2.2. Si $\hat{f}_{k} \in \Gamma\left(U, J_{k}\right)$, alors $\tilde{f}_{k} \mid V=0$.

DÉmonstration. Soit $x \in U$. Alors $\hat{f}_{k, x} \in J_{k, x}$ et on peut écrire:

$$
\hat{f}_{k, x}=\sum_{i=1}^{l}\left[f_{i}\left(T_{1}, \ldots, T_{p}\right)\left(\sum_{j=1}^{p} a_{i j} T_{j}\right)\right]
$$

avec $f_{i} \in O_{X, x}\left[T_{1}, \ldots, T_{p}\right], \operatorname{deg} f_{i}=k-1$ et $\left(a_{i 1}, \ldots, a_{i p}\right) \in H_{x}$ pour $i=1, \ldots, l$.

Pour tout $z \in \mathbf{C}^{p}$ tel que $(x, z) \in V$ on a donc

$$
\tilde{f}_{k,(x, z)}=\sum_{i=1}^{l}\left[f_{i}\left(z_{1}, \ldots, z_{p}\right)\left(\sum_{j=1}^{l} a_{i j} z_{j}\right)\right] \in I_{(x, z)}
$$

d'où $\left(\tilde{f}_{k} \mid V\right)_{(x, z)}=0$. Ceci vaut pour tout $(x, z) \in V$, par conséquent $\widetilde{f}_{k} \mid V=0$.

De la définition de $\sigma_{k}$ il suit immédiatement $\beta_{k} \circ \sigma_{k}=$ identité, donc la suite exacte (7) est bien scindée.

REMARQUE. Soit $T$ l'idéal qui définit la section nulle de $L(F)$ dans $L(F)$; pour tout entier positif $k, T^{k} / T^{k+1}$ est un faisceau cohérent sur $X$ (identifié à cette section nulle), et on a un isomorphisme canonique $T^{k} / T^{k+1} \stackrel{\sim}{\rightarrow} S^{k}(F)$. Pour le montrer, compte tenu de la suite exacte (7), il suffit de prouver que $\Gamma\left(V, T^{k} \mid V\right)=\Gamma\left(V, O_{Y}\right)_{k}$. 
Sur $U \times \mathbf{C}^{p}$ on a la suite exacte $0 \rightarrow I \cap T^{\prime k} \rightarrow T^{\prime k} \rightarrow T^{k} \rightarrow 0$ où $T^{\prime k}=$ $\left(z_{1}, \ldots, z_{p}\right) O_{U \times C} p$. Soit $f \in \Gamma\left(V, T^{k} \mid V\right)$. Il existe alors $\tilde{f} \in \Gamma\left(U \times \mathbf{C}^{p}, T^{\prime k}\right)$ qui étend $f$.

Il s'ensuit $\beta_{0}(f)=\ldots=\beta_{k-1}(f)=0$ et donc $f \in \Gamma\left(V, O_{Y}\right)_{k}$. Inversement soit $f \in \Gamma\left(V, O_{Y}\right)_{k}$. Il existe $\widetilde{f} \in \Gamma\left(U \times \mathbf{C}^{p}, O_{U \times \mathbf{C}}\right)$ qui étend $f$ et tel que dans son développement en série:

$$
\widetilde{f}(x, z)=\sum_{i_{1}, \ldots, i_{p}} \widetilde{f}_{i_{1}, \ldots, i_{p}}(x) z_{1}^{i_{1}} \ldots z_{p}^{i_{p}}
$$

on ait $\Sigma_{i_{1}+\ldots+i_{p}=l} \tilde{f}_{i_{1}, \ldots i_{p}, x} z_{1}^{i_{1}} \ldots z_{p}^{i_{p}} \in J_{l, x}$ pour $l=0,1, \ldots, k-1$ et pour tout $x \in U$. Donc pour $(x, z) \in V: \Sigma_{i_{1}}+\ldots+i_{p}=\widetilde{f}_{i_{1}}, \ldots, i_{p}, x z_{1}^{i_{1}} \ldots z^{i_{p}} \in I_{(x, z)}$, ce qui implique $\widetilde{f}_{(x, z)} \in I_{(x, z)}+T_{(x, z)}^{\prime k}$, i.e. $f_{(x, z)} \in T_{(x, z)}^{k}$ et $f \in \Gamma\left(V, T^{k} \mid V\right)$.

2. Soit maintenant $E$ un faisceau analytique cohérent sur $X$, qui admette sur $U$ une présentation:

$$
O_{X}^{s} \rightarrow O_{X}^{l} \rightarrow E \rightarrow 0
$$

En tensorisant cette présentation sur $O_{X}$ une fois par $O_{Y}$ et une fois par $S^{k}(F)$, et posant $\hat{E}=E \otimes O_{Y}$, on obtient les deux suites exactes: $O_{Y}^{s} \rightarrow O_{Y}^{l} \rightarrow \hat{E} \rightarrow 0$ et $\left(S^{k}(F)\right)^{s} \rightarrow\left(S^{k}(F)\right)^{l} \rightarrow E \otimes S^{k}(F) \rightarrow 0$ d'où, puisque $U$ et $V$ sont des espaces de Stein, en prenant les sections globales sur $V$ et $U$ respectivement, on obtient deux suites encore exactes:

$$
\begin{gathered}
\Gamma\left(V, O_{Y}^{s}\right) \rightarrow \Gamma\left(V, O_{Y}^{l}\right) \rightarrow \Gamma(V, \hat{E}) \rightarrow 0, \\
\Gamma\left(U,\left(S^{k}(F)\right)^{s}\right) \rightarrow \Gamma\left(U,\left(S^{k}(F)\right)^{l}\right) \rightarrow \Gamma\left(U, E \otimes S^{k}(F)\right) \rightarrow 0 .
\end{gathered}
$$

D'autre part on a un diagramme:

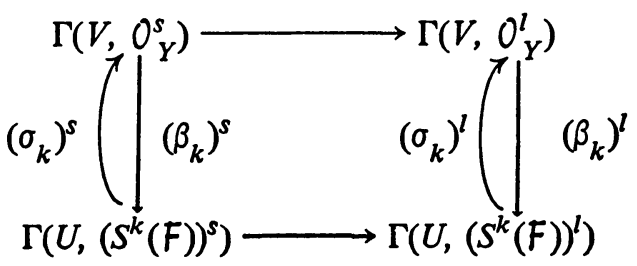

commutatif dans les deux sens. On en déduit qu'on peut définir deux applications linéaires $\gamma_{k}: \Gamma(V, \hat{E}) \rightarrow \Gamma\left(U, E \otimes S^{k}(F)\right)$ et $\eta_{k}: \Gamma\left(U, E \otimes S^{k}(F)\right) \rightarrow$ $\Gamma(V, \hat{E})$, telles que $\gamma_{k} \circ \eta_{k}=$ identité et que le diagramme suivant

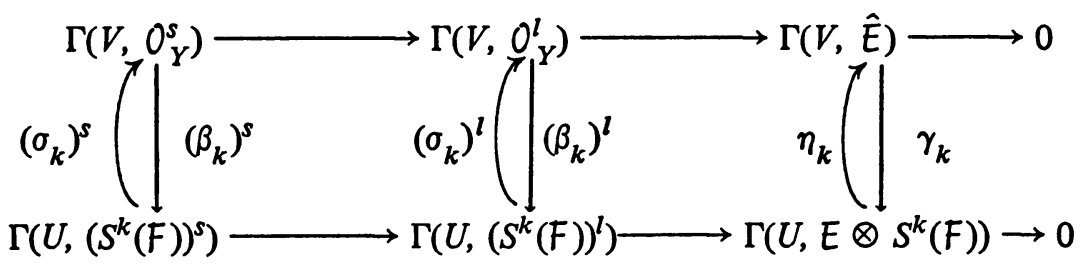

commute dans les deux sens. 
Les applications $\gamma_{k}$ et $\eta_{k}$ sont indépendantes du choix de la présentation de $E$ sur $U$. En effet si $O_{X}^{s^{\prime}} \rightarrow O_{X}^{l^{\prime}} \rightarrow E \rightarrow 0$ est un'autre présentation de $E$ sur $U$, on a un diagramme commutatif sur $U$ :

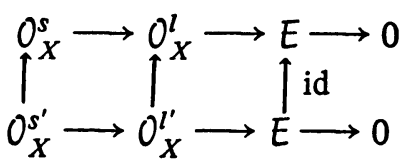

duquel on obtient un diagramme encore commutatif:

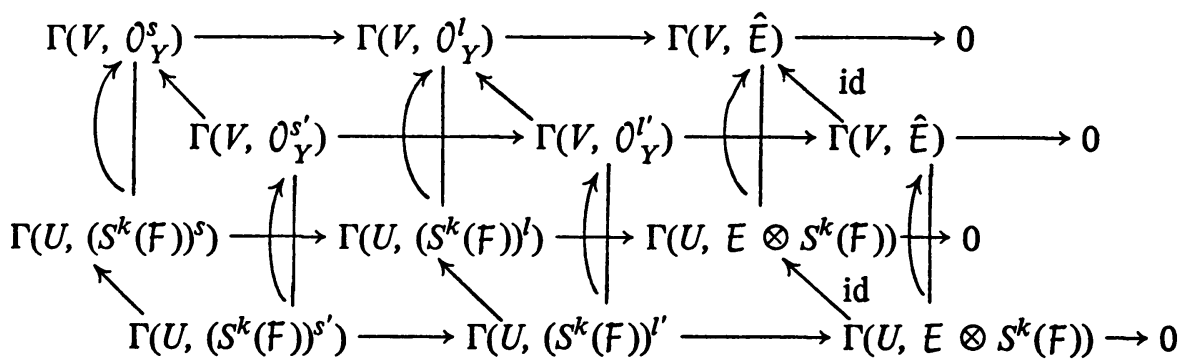

Notre affirmation en découle aisément.

Soit $\Gamma(V, \hat{E})_{k}=\left\{\sigma \in \Gamma(V, \hat{E}): \gamma_{0}(\sigma)=\ldots=\gamma_{k-1}(\sigma)=0\right\}$; on obtient une filtration de $\Gamma(V, \hat{E})$ et pour tout $k \geqslant 0$ une suite exacte scindée

$$
0 \rightarrow \Gamma(V, \hat{E})_{k+1} \rightarrow \Gamma(V, \hat{E})_{k} \frac{\eta_{k}}{\gamma_{k}} \Gamma\left(U, E \otimes S^{k}(F)\right) \rightarrow 0 .
$$

3. Soit maintenant $U=\left(U_{i}\right)_{i \in I}$ un recouvrement de $X$ formé d'ouverts de Stein tels que sur tout $U_{i}, F$ ait une résolution du type (1) et $E$ une présentation du type (8). En posant $V_{i}=L(F) \mid U_{i}$ on obtient un recouvrement de Stein $V=\left(V_{i}\right)_{i \in I}$ de $Y=L(F)$.

Considérons les groupes de cochaines

$$
C^{r}(V, \hat{E})=\prod_{i_{0}, \ldots, i_{r}} \Gamma\left(V_{i_{0}} \cap \ldots \cap V_{i_{r}} ; \hat{E}\right)
$$

sur tout facteur est définie une filtration; ceci induit une filtration $\left(C^{r}(V, \hat{E})_{k}\right)_{k \in \mathrm{N}}$ sur $C^{r}(V, \hat{E})$ compatible avec l'opérateur de cobord $\delta$. On a en outre une suite exacte scindée

$$
0 \rightarrow C^{r}(U, \hat{E})_{k+1} \rightarrow C^{r}(U, \hat{E})_{k} \leftrightharpoons C^{r}\left(U, E \otimes S^{k}(F)\right) \rightarrow 0
$$

La filtration sur les complexes de cochaines induit une filtration $\left(H^{r}(Y, \hat{E})_{k}\right)_{k \in \mathrm{N}}$ sur les groupes de cohomologie $H^{r}(Y, \hat{E})$ où $H^{r}(Y, \hat{E})_{k}$ est l'image des cocycles de $C^{r}(V, \hat{E})_{k} ; H^{r}(Y, \hat{E})_{k}$ est aussi l' $r$-ième groupe de cohomologie du complexe de cochaînes $\left\{C^{*}(V, \hat{E})_{k}, \delta\right\}$.

On obtient donc une suite exacte scindée: 


$$
0 \rightarrow H^{r}(Y, \hat{E})_{k+1} \rightarrow H^{r}(Y, \hat{E})_{k} \longmapsto H^{r}\left(X, E \otimes S^{k}(F)\right) \rightarrow 0
$$

De là on peut facilement conclure:

Proposition 2.3. Soient $X$ un espace complexe, $F$ un faisceau cohérent sur $X, Y=L(F)$ l'espace fibré linéaire associé. Pour tout faisceau cohérent $E$ sur $X$, posons $\hat{E}=E \otimes O_{Y}$. Alors:

(i) On a pour $r \in \mathrm{N}$ une filtration de $H^{r}(Y, \hat{E})$ telle que le groupe gradué associé soit isomorphe a $\bigoplus_{k \geqslant 0} H^{r}\left(X, E \otimes S^{k}(F)\right)$.

(ii) On à une injection naturelle: $\bigoplus_{k>0} H^{r}\left(X, E \otimes S^{k}(F)\right) \rightarrow H^{r}(Y, \hat{E})$.

3. Faisceaux faiblement positifs.

1. DÉFINITION 3.1. Soit $F$ un faisceau analytique cohérent sur un espace complexe $X$. On dit que $F$ est faiblement positif si la section nulle de l'espace fibré linéaire associé possède un voisinage ouvert 1-convexe.

Un fibré vectoriel $V$ sur $X$ est faiblement positif au sens de Grauert [6] si le faisceau des germes de sections holomorphes de $V$ est faiblement positif.

Le résultat suivant généralise [6, Hilfssatz $1, \S 3]$ :

THÉORÈmE 3.2. Soient $X$ un espace complexe, $F$ un faisceau faiblement positif sur $X$. Pour tout faisceau analytique cohérent $E$ sur $X$ il existe un entier positif $k_{0}=k_{0}(E)$ tel que $H^{r}\left(X, E \otimes S^{k}(F)\right)=0$ pour $k \geqslant k_{0}$ et $r \geqslant 1$.

Preuve. Soient $Y=L(F), T$ un voisinage 1-convexe de la section nulle de $Y$, et $\hat{E}=E \otimes O_{Y}$. Par composition de l'application $\bigoplus_{k>0} H^{r}\left(X, E \otimes S^{k}(F)\right)$ $\rightarrow H^{r}(Y, \hat{E})$ (Proposition 2.3(ii)) et de l'application de restriction $H^{r}(Y, \hat{E}) \rightarrow$ $H^{r}(T, \hat{E})$ on obtient un'application $\bigoplus_{k \geqslant 0} H^{r}\left(X, E \otimes S^{k}(F)\right) \rightarrow H^{r}(T, \hat{E})$. Si nous prouvons que cette application est injective, le théorème en suivra, puisque $T$ est 1 -convexe et $\operatorname{donc}_{\mathbf{c}} \operatorname{dim}_{\mathbf{C}} H^{r}(T, \hat{E})$ est finie.

Soient $\pi: Y \rightarrow X$ la projection, et $U=\left(U_{i}\right)_{i \in I}$ un recouvrement de Stein de $X$ tel que, en posant $V_{i}=\pi^{-1}\left(U_{i}\right)$ :

(*) Sur chaque $U_{i}$ on ait une présentation $O_{X}^{p_{i}} \rightarrow F \rightarrow 0$ de $F$ et donc un'immersion fermée $V_{i} \hookrightarrow U_{i} \times \mathrm{C}^{p_{i}}$.

(**) Pour tout $i \in I$ il existe un polydisque ouvert centré à l'origine $D_{i} \subset$ $\mathrm{C}^{p_{i}}$ tel que $T \supset W_{i}=\left(U_{i} \times D_{i}\right) \cap V_{i}$.

(***) Sur chaque $U_{i}$ on ait une présentation $O_{X}^{s_{i}} \rightarrow 0_{X}^{l_{i}} \rightarrow E \rightarrow 0$ de $E$.

Puisque $T$ est 1-convexe, pour tout $i V_{i} \cap T$ est un espace de Stein, donc $U \cap T=\left(V_{i} \cap T\right)_{i \in I}$ est un recouvrement de Stein de $T$.

Considérons d'abord le cas $E=O_{X}$, et prouvons que l'application composée

$$
\bigoplus_{k \geqslant 0} H^{r}\left(U, S^{k}(F)\right) \rightarrow H^{r}\left(V, O_{Y}\right) \rightarrow H^{r}\left(T, O_{Y}\right)
$$

est injective. Soit $f=\left(f_{i_{0}}, \ldots, i_{r}\right)_{i_{0}, \ldots, i_{r} \in I} \in Z^{r}\left(V, O_{Y}\right)$ tel qu'il existe $g=$ 
$\left(g_{j_{1}}, \ldots, j_{r}\right)_{j_{1}, \ldots, j_{r} \in I} \in C^{r}\left(V \cap T, O_{Y}\right)$ satisfaisant aux relations suivantes:

$$
f_{i_{0}, \ldots, i_{r}}\left|V_{i_{0}, \ldots, i_{r}} \cap T=\sum_{h=0}^{r}(-1)^{h+1} g_{i_{0}, \ldots, \hat{i}_{h}, \ldots, i_{r}}\right| V_{i_{0}, \ldots, i_{r}} \cap T
$$

$$
\text { (pour tout } \left.\left(i_{0}, \ldots, i_{r}\right) \in I^{r+1}\right) \text {, }
$$

et supposons que $f$ soit l'image d'un élément de $\bigoplus_{k \geqslant 0} Z^{r}\left(U, S^{k}(F)\right)$, c'est-à-dire qu'il existe des

$$
h_{k}=\left(h_{k, i_{0}, \ldots, i_{r}}\right)_{i_{0}, \ldots, i_{r} \in I} \in Z^{r}\left(U, S^{k}(F)\right) \quad(k=0, \ldots, n)
$$

tels qu'on ait:

$$
f_{i_{0}, \ldots, i_{r}}=\sigma_{0}\left(h_{0, i_{0}, \ldots, i_{r}}\right)+\ldots+\sigma_{n}\left(h_{n, i_{0}, \ldots, i_{r}}\right)
$$

$$
\text { (pour tout }\left(i_{0}, \ldots, i_{r}\right) \in I^{r+1} \text { ) }
$$

où les applications $\sigma_{0}, \ldots, \sigma_{n}$ sont celles définies au $\S 2.1$. Soit maintenant $U$ un ouvert de Stein de $X$ qui jouit des propriétés $(*)$ et $(* *)$, i.e. tel que sur $U$ il existe une présentation $O_{X}^{p} \rightarrow F \rightarrow 0$ de $F$, et qu'il existe un polydisque ouvert centré à l'origine $D \subset C^{p}$ tel que $T \supset W=(U \times D) \cap V$ (avec $V=$ $\left.\pi^{-1}(U)\right)$. Soit $g \in \Gamma\left(V \cap T, O_{Y}\right)$. Comme $W$ est un sous-ensemble analytique fermé de l'espace de Stein $U \times D$, il existe $\tilde{g} \in \Gamma\left(U \times D, O_{U \times \mathbf{C} p}\right)$ qui étend $g \mid W$. Soit

$$
\tilde{g}(x, z)=\sum_{j_{1}, \ldots, j_{p}} \tilde{g}_{j_{1}, \ldots, j_{p}}(x) z_{1}^{j_{1}} \ldots z_{p}^{j_{p}}
$$

le développement de $\widetilde{g}$ dans $U \times D$ par rapport aux coordonnés $z_{1}, \ldots, z_{p}$, et soit $\widetilde{g}_{k}=\Sigma_{j_{1}+\ldots+j_{p}=k} \widetilde{g}_{j_{1}}, \ldots, j_{p} z_{1}^{j_{1}} \ldots z_{p}^{j_{p}}$ pour tout entier positif $k$. Il est évident que $\widetilde{g}_{k} \in \Gamma\left(U \times \mathbf{C}^{p}, O_{U \times \mathbf{C}^{p}}\right)$; par suite on obtient un elément $g_{k}=$ $\widetilde{\alpha}_{k}\left(I_{k}\left(\widetilde{g_{k}}\right)\right) \in \Gamma\left(U, S^{k}(F)\right)$. Comme dans le $\S 2$ on voit facilement que l'élément $g_{k}$ ne dépend du choix ni de la présentation de $F$ sur $U$, ni de l'extension $\widetilde{g}$, ni du polydisque $D$.

Comme tout $U_{i_{0}, \ldots, i_{r}}=U_{i_{0}} \cap \ldots \cap U_{i_{r}}$ (resp. $U_{i_{1}, \ldots, j_{r}}$ ) possède les propriétés $(*),(* *)$, pour tout $k$ le procédé qui vient d'être décrit détermine $f_{i_{0}, \ldots, i_{r}, k} \in \Gamma\left(U_{i_{0}, \ldots, i_{r}}, S^{k}(F)\right)$ (resp. $g_{j_{1}, \ldots, j_{r}, k} \in \Gamma\left(U_{j_{1}, \ldots, i_{r}}, S^{k}(F)\right)$. On a

$$
f_{i_{0}, \ldots, i_{r} k}=\sum_{h=0}^{r} g_{i_{0}, \ldots, \hat{i}_{h}, \ldots, i_{r} k} \mid U_{i_{0}, \ldots, i_{r}} .
$$

En effet $g_{i_{0}, \ldots \hat{i}_{h}, \ldots, i_{r}, k} \mid U_{i_{0}, \ldots, i_{r}}$ peut se calculer de la manière suivante. Soit $g_{i_{0}, \ldots, i_{r}}^{h}=g_{i_{0}, \ldots, \hat{i}_{h}, \ldots, i_{r}} \mid W_{i_{0}, \ldots, i_{r}}$ et $\widetilde{g}_{i_{0}, \ldots, i_{r}}^{h}$ un'extension de $g_{i_{0}, \ldots, i_{r}}^{h}$ à $U_{i_{0}, \ldots, i_{r}} \times$ $D_{i_{0}, \ldots, i_{r}}$. Alors $g_{i_{0}, \ldots, \hat{i}_{h}, \ldots, i_{r}, k}=\widetilde{\alpha}_{k}\left(I_{k}\left(\widetilde{g}_{i_{0}}^{h}, \ldots, i_{r}, k\right)\right)$. Pour avoir la relation (11) il suffit de calculer $f_{i_{0}, \ldots, i_{r}, k}$ à partir de l'extension de $f_{i_{0}}, \ldots, i_{r} \mid W_{i_{0}, \ldots, i_{r}}$ donnée par

$$
\tilde{f}_{i_{0}, \ldots, i_{r}}=\sum_{h=0}^{r}(-1)^{h+1} \tilde{g}_{i_{0}, \ldots, i_{r}}^{h} .
$$


Pour conclure dans le cas $E=O_{X}$, compte tenu des (11), on n'a qu'à montrer que

$$
h_{k, i_{0}, \ldots, i_{r}}=f_{i_{0}, \ldots, i_{r}, k} \quad\left(i_{0}, \ldots, i_{r} \in I ; k=0, \ldots, n\right) .
$$

Mais ceci est une conséquence immédiate de la construction des applications $\sigma_{0}, \ldots, \sigma_{r}$ définies au $\S 2.1$ et des relations (10).

Considérons maintenant le cas général. Supposons que les (9) soient vraies avec $f \in Z^{r}(V, \hat{E})$ et $g \in C^{r}(V \cap T, \hat{E})$ et que de plus on ait

$$
f_{i_{0}, \ldots, i_{r}}=\eta_{0}\left(h_{0, i_{0}, \ldots, i_{r}}\right)+\ldots+\eta_{n}\left(h_{n, i_{0}, \ldots, i_{r}}\right)
$$

avec

$$
h_{k}=\left(h_{k, i_{0}, \ldots, i_{r}}\right) \in Z^{r}\left(E \otimes S^{k}(F)\right)(k=0, \ldots, n),
$$

où $\eta_{0}, \ldots, \eta_{n}$ sont les applications définies au $\S 2.2$.

Soit $U$ un ouvert de Stein de $X$ qui possède les propriétés $(*),(* *),(* * *)$ (on omet les indices). Pour tout entier positif $k$ on vient de définir des applications $\Gamma\left(W, O_{Y}\right) \rightarrow \Gamma\left(U, S^{k}(F)\right)$ d'où en procédant comme au $\$ 2.2$ on obtient des applications

$$
\Gamma(W, \hat{E}) \rightarrow \Gamma\left(U, E \otimes S^{k}(F)\right), \quad g \mapsto g_{k}
$$

qui rendent commutatif le diagramme suivant

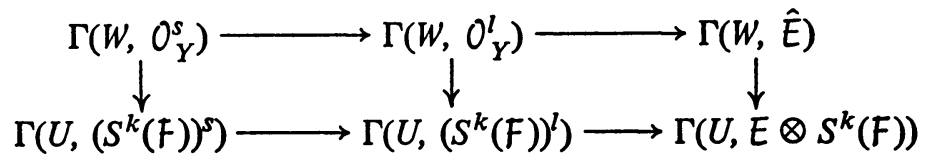

Ces applications sont indépendantes du choix de la présentation de $E$ sur $U$.

De la même manière du cas $E=O_{X}$ on trouve que les relations (11) et (12) avec $f_{i_{0}, \ldots, i_{r}, k} \in \Gamma\left(U_{i_{0}, \ldots, i_{r}}, E \otimes S^{k}(F)\right)$ et $g_{j_{1}, \ldots, j_{r}, k} \in \Gamma\left(U_{j_{1}}, \ldots, j_{r}, E \otimes S^{k}(F)\right)$ sont vraies.

La démonstration du Théorème 3.2 est ainsi achevée.

2. La Définition 3.1 peut se généraliser au cas relatif:

DÉfinition 3.3. Soit $p: X \rightarrow S$ un'application holomorphe entre deux espaces complexes, $F$ un faisceau analytique cohérent sur $X, L(F)$ l'espace fibré linéaire associé à $F, \pi: L(F) \rightarrow X$ la projection. On dit que $F$ est faiblement positif relativement à $S$ si pour tout $s \in S$ il existe un voisinage ouvert $U$ de $s$ et un voisinage ouvert $D$ de la section nulle de $L(F) \mid p^{-1}(U)$ tels que $p \circ \pi \mid D: D$ $\rightarrow X$ soit un'application 1-convexe.

On a le

THÉORÈME 3.4. Soient $p: X \rightarrow S$ un'application holomorphe propre entre deux espáces complexes, $F$ un faisceau sur $X$ faiblement positif relativement à $S$, 
$K$ un compact de $S$. Pour tout faisceau cohérent $E$ sur $X$ il existe un entier positif $k_{0}=k_{0}(E)$ tel que sur $K$ on ait: $R^{n} p_{*}\left(E \otimes S^{k}(F)\right)=0$ pour $k \geqslant k_{0}$ et $n \geqslant 1$.

DÉmonstration (CF. [7, ThEOREM 6.2]). Soit $N$ la section nulle de $L(F)$, soit $\pi: L(F) \rightarrow X$ la projection, et fixons $n \geqslant 1$. On peut supposer qu'il existe un voisinage ouvert $D$ de $N$ tel que $p \circ \pi \mid D$ soit un'application 1-convexe. Pour tout $r \geqslant 1$ et tout ouvert $U \subset S$ on a des injections (Proposition 2.3):

$$
H^{n}\left(p^{-1}(U), \bigoplus_{k=0}^{r}\left(E \otimes S^{k}(F)\right)\right) \rightarrow H^{n}\left((p \circ \pi)^{-1}(U) \cap D, \pi^{*} E\right) .
$$

Il s'ensuit que pour $k \geqslant 1$ les applications

$$
R^{n} p_{*}\left(\bigoplus_{k=0}^{r}\left(E \otimes S^{k}(F)\right)\right) \rightarrow R^{n}(p \circ \pi \mid D)_{*}\left(\pi^{*} E\right)
$$

sont aussi injectives. Le faisceau $R^{n}(p \circ \pi \mid D)_{*}\left(\pi^{*} E\right)$ est cohérent parce que $p \circ \pi \mid D$ est 1 -convexe, et $R^{n} p_{*}\left(E \otimes S^{k}(F)\right)$ est cohérent pour tout $k \geqslant 1$ parce que $p$ est propre. Soit

$$
H_{r}=\bigoplus_{k=0}^{r} R^{n} p_{*}\left(E \otimes S^{k}(F)\right)
$$

Grace aux injections (13) on peut regarder les $H_{r}$ comme une suite croissante de sous-faisceaux cohérents de $R^{n}(p \circ \pi \mid D)_{*}\left(\pi^{*} E\right)$; comme $K$ est compact, cette suite doit être stationnaire sur $K$, ce qui implique le théorème.

4. Généralisation d'un théorème de Kodaira et Grauert.

1. Le théorème suivant est une généralisation faible d'un théorème dû à Kodaira [8] et Grauert [6].

THÉORÈme 4.1. Soient $X$ un espace complexe compact réduit et $F$ un faisceau faiblement positif sur $X$, tel que Supp $F=X$. Alors $X$ est un espace de Moišezon.

DÉmonstration. Il est évident qu'on peut supposer $X$ irréductible. Pour $x \in X$ soit $M_{x}^{2}$ le faisceau de germes de fonctions holomorphes sur $X$ ayant en $x$ un zéro au moins du second ordre. On a une suite exacte

$$
0 \rightarrow M_{x}^{2} \rightarrow \mathrm{O}_{X} \rightarrow \mathrm{O}_{X} / M_{x}^{2} \rightarrow 0 \text {. }
$$

Soit $k_{0} \in \mathrm{N}$ tel que $H^{1}\left(X, M_{x}^{2} \otimes S^{k} 0(F)\right)=0$ (Théorème 3.2). En tensorisant la suite exacte précédente par $S^{k} 0(F)$ on obtient la suite exacte:

$$
\begin{aligned}
& 0 \rightarrow \operatorname{Tor}_{1}^{0} X\left(O_{X} / M_{x}^{2}, s^{k} 0(F)\right) \rightarrow M_{x}^{2} \otimes S^{k} 0(F) \\
& \stackrel{\alpha}{\rightarrow} S^{k} 0(F) \rightarrow O_{X} / M_{x}^{2} \otimes S^{k}(F) \rightarrow 0
\end{aligned}
$$


et de celle-ci deux suites exactes courtes:

$$
\begin{gathered}
0 \rightarrow \operatorname{Tor}_{1}^{0} X\left(O_{X} / M_{x}^{2}, s^{k} 0(F)\right) \rightarrow M_{x}^{2} \otimes S^{k} 0(F) \rightarrow \operatorname{Ker} \alpha \rightarrow 0, \\
0 \rightarrow \operatorname{Ker} \alpha \rightarrow s^{k} 0(F) \rightarrow O_{X} / M_{x}^{2} \otimes s^{k} 0(F) \rightarrow 0 .
\end{gathered}
$$

De la première on a une suite exacte de cohomologie:

$$
0=H^{1}\left(X, M_{x}^{2} \otimes S^{k} 0(F)\right) \rightarrow H^{1}(X, \operatorname{Ker} \alpha) \rightarrow H^{2}\left(X, \text { Tor }_{1}^{0} X\left(O_{X} / M_{x}^{2}, s^{k} 0(F)\right)\right) \text {. }
$$

Comme le support de $O_{X} / M_{x}^{2}$ est réduit à $\{x\}$, se réduit à $\{x\}$ aussi le support de $\operatorname{Tor}_{1}^{O_{x}}\left(O_{X} \mid M_{x}^{2}, S^{k} 0(F)\right)$. Il s'ensuit $H^{2}\left(X, \operatorname{Tor}_{1}^{0_{X}}\left(O_{X} / M_{x}^{2}, S^{k} 0(F)\right)\right)=0$, et par suite $H^{1}(X, \operatorname{Ker} \alpha)=0$.

De la deuxième suite exacte courte on obtient alors un'application surjective

$$
\Gamma\left(X, S^{k} 0(F)\right) \rightarrow\left(O_{X} / M_{x}^{2} \otimes S^{k} 0(F)\right)_{x} .
$$

Le nombre $k_{0}$ ci-dessus dépend du point $x \in X$; le lemme suivant nous assûre qu'il existe un voisinage de $x$ tel que l'application (14) soit encore surjective quand on remplace $x$ par un point de ce voisinage.

LEMme 4.2. Soient $X$ un espace complexe compact, $S$ un faisceau analytique cohérent sur $X$. L'ensemble des points $x \in X$ où l'application canonique $\Gamma(X, S) \rightarrow\left(O_{X} / M_{x}^{2} \otimes S\right)_{x}$ 'est pas surjective est un. sous-ensemble analytique fermé de $X$.

Preuve du lemme. Soit $Z$ l'ensemble en question. Si $P_{X}^{1}$ est le faisceau des parties principales d'ordre 1 sur $X$ (v. [4, exp. 15]), on a (ibid., Corollaire 2.5) un isomorphisme: $P_{X}^{1} \otimes \mathrm{C} \simeq\left(O_{X} / M_{x}^{2}\right)_{x}$.

Considérons le morphisme de faisceaux $O_{X} \rightarrow P_{X}^{1}$ qui définit la structure d' $O_{X}$-algèbre de $P_{X}^{1}$. En tensorisant par $S$ et en prenant les sections globales on obtient un'application linéaire $\Gamma(X, S) \rightarrow \Gamma\left(X, P_{X}^{1} \otimes S\right)$ qui rend commutatif le diagramme suivant:

$$
\begin{aligned}
\Gamma(X, S) & \longrightarrow \Gamma\left(X, P_{X}^{1} \otimes S\right) \\
\downarrow & \downarrow \\
\left(O_{X} / M_{x}^{2} \otimes S\right)_{x} & \longrightarrow P_{X}^{1} \otimes S \otimes C .
\end{aligned}
$$

Soient $\sigma_{1}, \ldots, \sigma_{p}$ une base de $\Gamma(X, S)$ et $h_{1}, \ldots, h_{p}$ leurs images dans $\Gamma\left(X, P_{X}^{1} \otimes S\right)$. Si $H$ est le sous-faisceau de $P_{X}^{1} \otimes S$ engendré $\operatorname{par} h_{1}, \ldots, h_{p}$ on trouve qu'on a $Z=\operatorname{Supp}\left(P_{X}^{1} \otimes S / H\right)$ par une simple application de lemme de Nakayama. Ceci prouve le lemme.

Posons maintenant $A=\mathrm{C}\left\{y \in X: F_{y}\right.$ est libre sur $\left.\mathrm{O}_{X, y}\right\}$; c'est un sous-ensemble analytique de $X$. Soient $\sigma_{1}, \ldots, \sigma_{h}$ une base de $\Gamma\left(X, S^{k}(F)\right)$. La surjectivité de l'application (14) et le Lemme 4.2 impliquent qu'il existe un 
voisinage $U$ de $x$ tel que $\sigma_{1}, \ldots, \sigma_{h}$ donnent des coordonnés locales aux points de $U A .\left(^{3}\right)$ En outre pour tout multiple $k=n k_{0}$ de $k_{0}$ les sections de $\Gamma\left(X, S^{k}(F)\right)$ données par $\sigma_{1}^{i_{1}} \ldots \sigma_{h}^{i_{h}}$ avec $i_{1}+\ldots+i_{h}=n$ donnent encore des coordonnés locales sur $U \backslash A$.

Soient maintenant $x_{1}, x_{2}$ deux points de $X$, et soit $M_{x_{1} x_{2}}$ le faisceau des germes de fonctions holomorphes sur $X$ nulles en $x_{1}$ et $x_{2}$. Soit $k_{1}$ tel que $H^{1}\left(X, M_{x_{1} x_{2}} \otimes S^{k_{1}}(F)\right)=0$ (Théorème 3.2). De la suite exacte

$$
0 \rightarrow M_{x_{1} x_{2}} \rightarrow \mathrm{O}_{X} \rightarrow \mathrm{O}_{X} / M_{x_{1} x_{2}} \rightarrow 0
$$

avec le même procédé que ci-dessus on obtient un'application surjective

$$
\Gamma\left(X, S^{k}(F)\right) \rightarrow \Gamma\left(X, O_{X} / M_{x_{1} x_{2}} \otimes S^{k}(F)\right) \text {. }
$$

On a

$$
\begin{aligned}
\Gamma\left(X, O_{X} / M_{x_{1} x_{2}} \otimes S^{k}(F)\right)= & \left(O_{X, x_{1}} / M_{x_{1}} \otimes_{O_{X, x_{1}}}\left(S^{k}(F)\right)_{x_{1}}\right) \\
& \oplus\left(O_{X, x_{2}} / M_{x_{2}} \otimes_{O_{X, x_{2}}}\left(S^{k}(F)\right)_{x_{2}}\right)
\end{aligned}
$$

où $m_{x_{1}}$ (resp. $m_{x_{2}}$ ) est l'idéal maximal de $O_{X, x_{1}}$ (resp. $O_{X, x_{2}}$ ). Une simple application du lemme de Nakayama montre qu'il existe deux voisinages, $U_{1}$ de $x_{1}$ et $U_{2}$ de $x_{2}$ tels que pour $x_{1}^{\prime} \in U_{1}$ et $x_{2}^{\prime} \in U_{2}$ on a une surjection du type (15).

Par suite il existe un nombre fini de sections globales de $S^{k_{1}}(F), \alpha_{1}, \ldots, \alpha_{l}$, qui séparent les points de $U_{1} \backslash A$ et $U_{2} \backslash A .\left({ }^{4}\right) \mathrm{Si} k^{\prime}=n k_{1}$, une propriété analogue est vraie pour $S^{k^{\prime}}(F)$.

Comme $X$ est compact, on peut trouver un nombre fini $s_{1}, \ldots, s_{q}$ de sections globales d'un certain $S^{t}(F)$ qui séparent les points et donnent des coordonnés locales sur $X \backslash A$, et telles que le morphisme de faisceaux $O_{X}^{q} \rightarrow S^{t}(F)$ défini par $s_{1}, \ldots, s_{q}$ soit surjectif.

Appliquons maintenant le Théorème 1.2. Soit donc $\pi: \widetilde{X} \rightarrow X$ une modification propre telle que $\pi \mid \widetilde{X} \backslash \pi^{-1}(A)$ soit un isomorphisme et $E=$ $\pi^{*} S^{t}(F) \mid T\left(\pi^{*} S^{t}(F)\right)$ soit un faisceau localement libre, de rang $l$ égal au rang de $S^{t}(F) \mid X \backslash A$ (et donc non nul parce que Supp $\left.F=X\right)$. On a un morphisme surjectif $O_{X}^{q} \rightarrow E$ défini par des sections $\tilde{s}_{1}, \ldots, \tilde{s}_{q} \in \Gamma(X, E)$ d'où un'immersion fermée:

(3) Les restrictions de $\sigma_{1}, \ldots, \sigma_{h}$ à $X \backslash A$ déterminent un morphisme de faisceaux $\left.\left.0_{X}^{h}\right|_{X \backslash A} \rightarrow S^{k} 0(F)\right|_{X \backslash A}$. Si $r$ est le rang de $S^{k} 0(F)$, on obtient un application holomorphe

$$
\operatorname{Grass}_{r}\left(\left.S^{k^{0}}(F)\right|_{X \backslash A}\right)=X \backslash A \rightarrow \text { Grass }_{r}\left(\left.0_{X}^{h}\right|_{X \backslash A}\right)=(X \backslash A) \times G(h-r, h)
$$

qui, composée avec la projection, donne un'autre application $u: X \backslash A \rightarrow G(h-r, h)$. L'expression " $\sigma_{1}, \ldots, \sigma_{h}$ donnent des coordonnés locales aux points de $U \backslash A$ " signifie que cette dernière application est un'immersion en tout point de $U \backslash A$.

(4) Ceci signifie qu'on a $u\left(U_{1} \backslash A\right) \cap u\left(U_{2} \backslash A\right)=\varnothing, u$ étant l'application définie comme dans la note précédente à partir des sections $\alpha_{1}, \ldots, \alpha_{l}$. 


$$
\widetilde{X}=\operatorname{Grass}_{l} E \hookrightarrow \operatorname{Grass}_{l}\left(O_{\widetilde{X}}^{q}\right)=\widetilde{X} \times G(q-l, q) .
$$

En composant avec la projection on obtient un'application $\psi: \widetilde{X} \rightarrow$ $G(q-l, q)$. Comme $\widetilde{X}$ est compact, $\psi(\widetilde{X})$ est un sous-espace analytique fermé

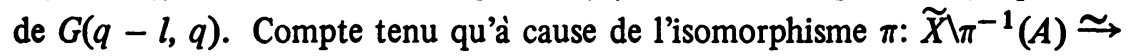
$X \backslash A$ les sections $\widetilde{s_{1}}, \ldots, \widetilde{s}_{q}$ donnent des coordonnés locales et séparent les points sur $\widetilde{X} \backslash \pi^{-1}(A)$, on voit facilement que $\psi$ est un'application holomorphe de $\widetilde{X}$ sur $\psi(\widetilde{X})$ dont la réciproque est méromorphe au sens de Remmert [9]. Comme $\psi(\widetilde{X})$ est un espace de Moišezon, $\widetilde{X}$ (et donc $X$ ) l'est aussi. C.Q.F.D.

\section{Démontrons finalement le}

THÉORÈmE 4.3. Soient $X$ un espace complexe compact réduit, $F$ un faisceau analytique cohérent sur $X$, et $A=\mathrm{C}\left\{x \in X: F_{x}\right.$ est libre sur $\left.O_{X, x}\right\}$. Si l'algèbre $A(F)=\bigoplus_{k \geq 0} \Gamma\left(X, S^{k}(F)\right)$ sépare les points et donne des coordonnés locales sur $X \backslash A, X$ est un espace de Moišezon.

On peut supposer $X$ irréductible. De plus, grâce au Théorème 1.2 on peut supposer que $F$ soit localement libre et que $A(F)$ donne des coordonnés locales et sépare les points en dehors d'un sous-ensemble analytique maigre $A$ de $X$. Etant donné des sections $\sigma_{1}, \ldots, \sigma_{n} \in \Gamma\left(X, S^{k}(F)\right)$ notons $\Sigma\left(\sigma_{1}, \ldots, \sigma_{n}\right)$ l'ensemble des points de $X$ où $\sigma_{1}, \ldots, \sigma_{n}$ ne donnent pas des coordonnés locales, et $Z\left(\sigma_{1}, \ldots, \sigma_{n}\right)$ l'ensemble des couples $\left(x_{1}, x_{2}\right) \in X \times X$ tels que $\sigma_{1}, \ldots, \sigma_{n}$ ne séparent pas $x_{1}$ et $x_{2}$; ce sont des sous-ensembles analytiques de $X$ et $X \times X$ respectivement.

Soient $\sigma_{1}, \ldots, \sigma_{n}$ des sections globales d'un certain $S^{k}(F)$ qui donnent des coordonnés locales en quelque point de $X$, et soient $Z_{1}, \ldots, Z_{s}$ les composantes irréductibles de $\Sigma\left(\sigma_{1}, \ldots, \sigma_{n}\right)$ non incluses dans $A$. Soient $x_{1} \in$ $Z_{1} \backslash A, \ldots, x_{s} \in Z_{s} \backslash A$. Il existe des sections globales $\sigma_{1}^{\prime}, \ldots, \sigma_{t}^{\prime}$ d'un $S^{k_{1}}(F)$ convenable qui donnent des coordonnés locales en $x_{1}, \ldots, x_{s}$. Si on pose

$$
\begin{array}{ll}
\sigma_{i_{1}, \ldots, i_{n}}=\sigma_{1}^{i_{1}} \ldots \sigma_{n}^{i_{n}} & \left(i_{1}+\ldots+i_{n}=k_{1}\right), \\
\sigma_{j_{1}}^{\prime}, \ldots, j_{t}=\sigma_{1}^{\prime} \ldots \sigma_{t}^{j_{t}} & \left(j_{1}+\ldots+j_{t}=k_{0}\right)
\end{array}
$$

et on note $Z_{1}^{\prime}, \ldots, Z_{m}^{\prime}$ les composantes irréductibles de $\Sigma\left(., \sigma_{i_{1}}, \ldots, i_{n}, \ldots, \sigma_{j_{1}}^{\prime}, \ldots, j_{t}, \ldots\right)$ qui ne sont pas incluses dans $A$, on aura $\operatorname{dim}_{\mathrm{C}}\left(\bigcup_{i=1}^{m} Z_{i}^{\prime}\right)<\operatorname{dim}_{\mathrm{C}}\left(\bigcup_{j=1}^{s} Z_{j}\right)$. Il est clair alors qu'ainsi poursuivant on pourra trouver un nombre fini de sections globales d'un $S^{k}(F)$ convenable qui donnent des coordonnés locales sur $X \backslash A$.

Avec un raissonement analogue, remplaçant $X$ par $X \times X$ et $\Sigma\left(\sigma_{1}, \ldots, \sigma_{n}\right)$ par $Z\left(\sigma_{1}, \ldots, \sigma_{n}\right)$ on peut affirmer qu'il existe un nombre fini de sections 
globales d'un certain $S^{h}(F)$ qui séparent les points de $X \backslash A$.

On peut donc conclure qu'il existe des sections globales, en nombre fini, d'un $S^{t}(F)$ convenable, qui séparent les points et donnent des coordonnés locales sur $X \backslash A$. En procédant comme dans la partie finale de la démonstration du Théorème 4.1, on conclut que $X$ est un espace de Moišezon.

\title{
BIBLIOGRAPHIE
}

1. V. Ancona, Fasci debolmente positivi su uno spazio complesso, Atti Accad. Naz. Lincei Rend. Cl. Sci. Fis. Mat. Natur. (8) 54 (1973), 567-569.

2. A. Andreotti and $\mathrm{H}$. Grauert, Théorèmes de finitude pour la cohomologie des espaces complexes, Bull. Soc. Math. France 90 (1962), 193-259. MR 27 \#343.

3. A. Andreotti and G. Tomassini, A remark on the vanishing of certain cohomology groups, Compositio Math. 21 (1969), 417-430. MR 41 \#5651.

4. Séminaire Henri Cartan 13ième année: 1960/61, Familles d'espaces complexes et fondements de la géométrie analytique, fasc. 1,2, École Normale Supérieure, Secrétariat mathématique, Paris, 1962. MR 26 \#3562.

5. G. Fischer, Lineare Faserräume und kohärente Modulgarben über komplexen Räumen, Arch. Math. (Basel) 18 (1967), 609-617. MR 36 \#4024.

6. H. Grauert, Uber Modifikationen und exzeptionelle analytische Mengen, Math. Ann. 146 (1962), 331-368. MR 25 \#583.

7. K. Knorr and M. Schneider, Relativexzeptionelle analytische Mengen, Math. Ann. (1971), 238-254. MR 45 \#2208.

8. K. Kodaira, On Kähler varieties of restricted type (an intrinsic characterization of algebraic varieties), Ann. of Math. (2) 60 (1954), 28-48. MR 16, 952.

9. R. Remmert, Holomorphe und meromorphe Abbildungen komplexer Räume, Math. Ann. 133 (1957), 328-370. MR 19, 1193.

10. O. Riemenschneider, Characterizing Moǐsezon spaces by almost positive coherent analytic sheaves, Math. Z. 123 (1971), 263-284. MR 45 \#3782.

11. A generalization of Kodaira's embedding theorem, Math. Ann. 200 (1973), 99-102. MR 48 \#4355.

12. H. E. Rossi, Picard variety of an isolated singular point, Rice Univ. Studies 54 (1968), no. 4, 63-73. MR 39 \#5831.

\author{
ISTITUTO MATEMATICO DELL'UNIVERSITA, VIA SAVONAROLA 9. 44100 \\ FERRARA, ITALIE
}

